\title{
Temporomandibular Joint Replacement Device Research Wear and Corrosion Technology Transfer from Orthopedics
}

\author{
L. G. Mercuri • M. T. Mathew $\cdot$ S. Kerwell • \\ H. Lundberg • C. Sukotjo
}

Received: 27 August 2014/Revised: 7 October 2014/Accepted: 16 October 2014/Published online: 28 October 2014

(c) Springer International Publishing AG 2014

\begin{abstract}
The general requirements for joint replacement devices emphasizes the importance of device material biocompatibility, with no inflammatory or toxic response to wear beyond a tolerable level, the appropriate mechanical properties for the desired application, and lastly economically viable manufacturing and processing methods. Implicit in these requirements is the importance of understanding wear and failure mechanisms of implanted devices. However, compared to orthopedic total joint replacement (TJR) devices, functional wear failure mechanisms for temporomandibular joint (TMJ) TJR implants have not been clearly defined. Our research group has started initial translational investigations involving the analysis of failed retrieved TMJ TJR devices alloy microstructure compared to control, never implanted, TMJ TJR devices utilizing established orthopedic TJR device retrieval tribocorrosion evaluation protocols. This and future studies will guide future material choices and functional design improvements for TMJ TJR devices. Orthopedic TJR implant schemes may also be improved by understanding the degradation mechanism of TMJ TJR
\end{abstract}

L. G. Mercuri $(\bowtie) \cdot$ M. T. Mathew · S. Kerwell · H. Lundberg Department of Orthopedic Surgery, Rush University Medical Center, Chicago, USA

e-mail: lgm@tmjconcepts.com

L. G. Mercuri · M. T. Mathew - S. Kerwell · C. Sukotjo Institute of Biomaterials, Tribocorrosion, and Nanomedicine (IBTN), Chicago, USA

M. T. Mathew · S. Kerwell

Department of Bioengineering, University of Illinois at Chicago, Chicago, USA

M. T. Mathew · C. Sukotjo

Department of Restorative Dentistry, University of Illinois at Chicago, Chicago, USA implants, as the materials employed in both TJR devices are similar.

Keywords Tribocorrosion - Temporomandibular joint replacement

The human temporomandibular joint (TMJ) is the diarthroidial articulation of the mandible to the temporal bone. The essential life functions of mastication, speech, airway support, and deglutition are supported by TMJ function and form. This places the TMJ complex under more cyclical loading and unloading than any other body joint over a lifetime [1].

End-stage disease is the very worst condition of an organ or disease state. At this point, the organ is barely functioning. Examples include end-stage kidney disease where the kidneys have shut down and the patient requires dialysis, or endstage cardiac disease which means the heart is functioning very poorly and may need mechanical support or transplantation in order for the patient to survive.

Applying this term to joints, end-stage joint disease connotes a joint that is so negatively affected architecturally by disease or injury that it results in severe functional impairment for the patient.

As with all other joints, the TMJ is affected by all of the end-stage joint diseases resulting from developmental disorders, neoplasia, trauma, arthritic disease, failed prior joint surgery, or ankylosis, and require replacement. Therefore, to provide long-term safe and effective outcomes, any TMJ total joint replacement (TJR) device must be able to manage the anatomical, operational, and esthetic discrepancies presented, withstand the stresses and strains of functional loading, while exhibiting minimal wearrelated concerns [2]. 
Fig. 1 a Stock metal-onpolyethylene device (Biomet Microfixation, Jacksonville, FL); b Stock metal-on-metal device (Nexus CMF, Salt Lake City, UT); c Custom metal-onmetal device (Nexus CMF, Salt Lake City, UT); d Patient-fitted metal-on-polyethylene TMJ Concepts device (TMJ Concepts, Ventura, CA-image credit-Hunterian Museum, Royal College of Surgeons of England, London, UK)
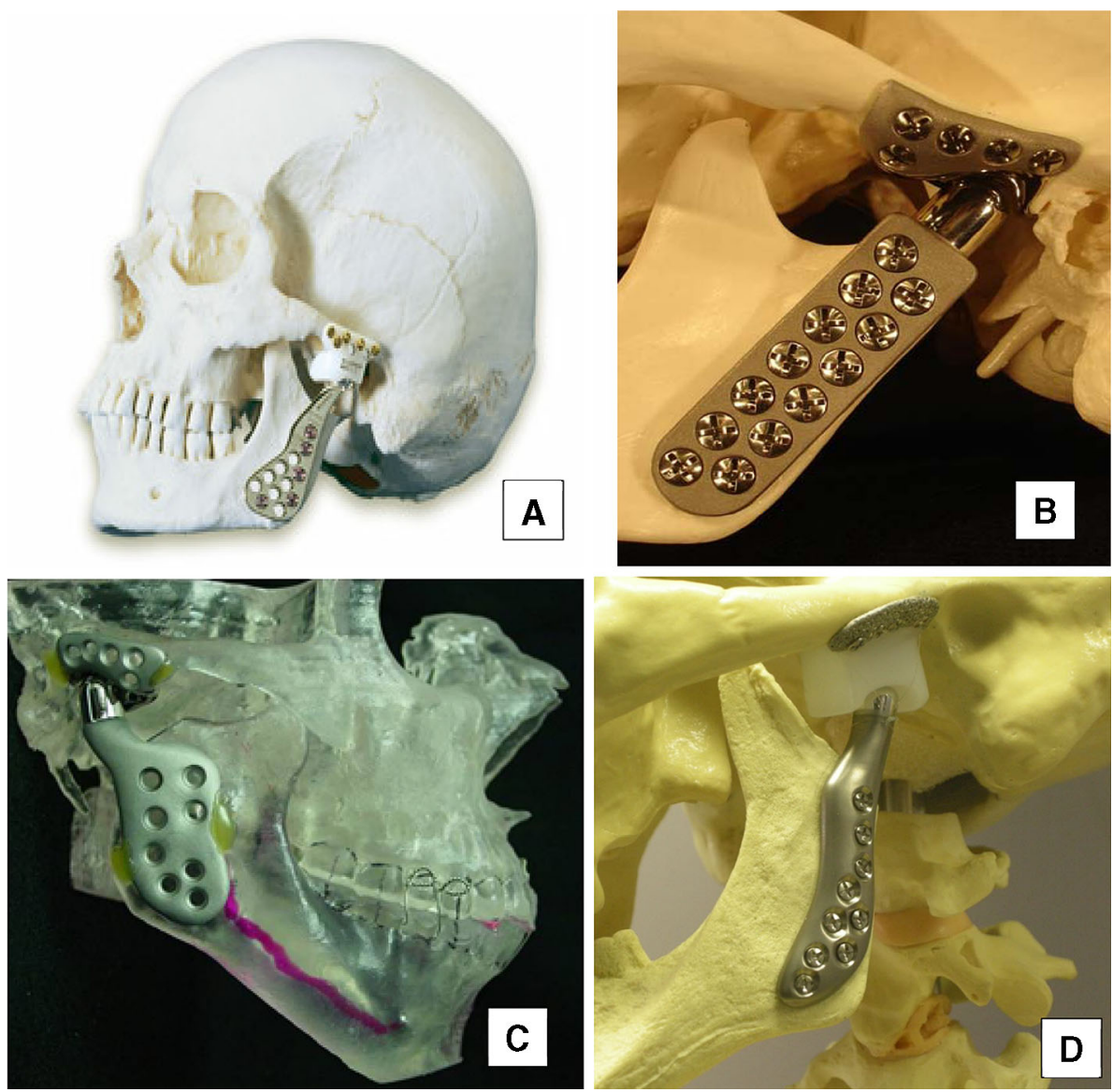

There are two categories of TMJ TJR devices approved by the Food and Drug Administration for use in the United States. First are stock or "off-the-shelf" devices, which the surgeon has to make fit at implantation (Biomet Microfixation TMJ Replacement System, Jacksonville, FL, USA; Nexus CMF, Salt Lake City, UT, USA). Second are custom or "patient-fitted" devices which are made to fit each specific case utilizing CAD/CAM technology (TMJ Concepts, Ventura, CA, USA; Nexus CMF, Salt Lake City, UT, USA) (Fig. 1).

In the orthopedic literature, it has become evident that some THR failures are related to material breakdown due to wear and corrosion leading to metal ion release. The overall poor performance of such joints is the result of a local and/or system response to the combined effect of both wear and corrosion [3-8].

In a description of the general requirements for joint replacement devices, Katti emphasized the importance of device material biocompatibility, with no inflammatory or toxic response to wear beyond a tolerable level, the appropriate mechanical properties for the desired application, and lastly economically viable manufacturing and processing methods [9].
Implicit in these requirements is the importance of understanding wear and failure mechanisms of implanted devices. However, compared to orthopedic TJR devices, functional wear failure mechanisms for TMJ TJR implants have not been clearly defined. This may be because there are exponentially fewer TMJ TJR devices implanted annually than orthopedic TJR devices. What is understood is that not only must wear and corrosion influence TJR implant in vivo longevity, but also peri-implant tissue reactivity to these two phenomena.

Our research group has started initial translational investigations involving the analysis of failed retrieved TMJ TJR devices alloy microstructure compared to control, never implanted, TMJ TJR devices utilizing established orthopedic TJR device retrieval wear, and corrosion evaluation protocols.

Thirty-one failed TMJ TJR samples were obtained from two independent sources, a group of international TMJ surgeons and the retrieval collection at the TMJ Implant Registry and Repository located at the University of Minnesota. The inventory was comprised three failed retrieved TMJ TJR groups of devices: Group 1-Metal-on-metal (MoM): consisted of 19 failed retrieved metal (CoCrMo) 
condyle-on-metal (CoCrMo) fossa devices. Group 2Polymethymethacrylate-on-metal (PoM): consisted of a total of 7 failed retrieved polymethylmethacrylate (PMMA) condyle-on-metal ( $\mathrm{CoCrMo}$ ) fossa devices. Group 3 consisted of 2 titanium-nitride (TiN)-coated condyle-on-fossa devices. In addition to the study failed retrieved TMJ TJR devices, a control Group 4, consisting of 3 never implanted MoM-type CoCrMo TMJ TJR devices, was included.

Microscopic images using the SmartScope automatic measuring system, white light interferometry (WLI), scanning electron microscopy (SEM), and Raman spectroscopy were conducted to characterize the surfaces of the device glenoid fossa (cup) and mandibular condyle (ball) components in each group where applicable.

Substantial surface damage was observed in the articulating areas between the condyle and the fossa components in all retrieval Groups. Damage included pitting corrosion, evidence of deposited corrosion products, specific wear patterns, hard phases, surface depressions, and bi-directional scratches, which are similar to the findings of metalon-metal failed retrieved THR devices.

Electrochemical testing was performed on Group 1 and 3 retrieval samples and the Group 4 control. Electrochemical impedance spectroscopy (EIS) confirmed material properties as well as corrosion kinetics in vivo help mitigate corrosion as reflected by the Raman spectroscopy results.

In summary, this study demonstrated the potential role of wear and corrosion interactions on the failure of TMJ TJR devices. Further studies will include in vitro investigation of corrosion kinetics and the synergistic interactions of wear and corrosion and their specific roles in the underlying tribocorrosion mechanisms of TMJ TJR devices. The generation and distribution of corrosion products and wear particles due to the tribocorrosion processes will also be considered as it has several clinical implications and potentials sources of many side effects on the patients [10,11].

This and future studies will guide future material choices and functional design improvements for TMJ TJR devices. Orthopedic TJR implant schemes may also be improved by understanding the degradation mechanism of TMJ TJR implants, as the materials employed in both TJR devices are similar.

Conflict of interest On behalf of all authors, the corresponding author states that there is no conflict of interest.

\section{References}

1. Ingawale S, Goswami T (2009) Temporomandibular joint: disorders, treatments, and biomechanics. Ann Biomed Eng 37:976-996

2. Mercuri LG (2008) Temporomandibular joint reconstruction. In: Fonseca R (ed) Oral and maxillofacial surgery. Elsevier, Philadelphia, pp 945-960 [Chapter 51]

3. Bozic KJ, Kurtz SM, Lau E, Ong K, Vail TP, Berry DJ (2009) The epidemiology of revision total hip arthroplasty in the United States. J Bone Joint Surg 91:128-133

4. Bozic KJ, Kurtz S, Lau E, Ong K, Chiu V, Vail TP et al (2009) The epidemiology of bearing surface usage in total hip arthroplasty in the United States. J Bone Joint Surg 91:1614-1620

5. Wilson NA, Schneller ES, Montgomery K, Bozic KJ (2008) Hip and knee implants: current trends and policy considerations. Health Aff (Millwood). 27:1587-1598

6. Kop AM, Swarts E (2009) Corrosion of a hip stem with a modular neck taper junction: a retrieval study of 16 cases. J Arthroplasty 24:1019-1023

7. Gilbert JL, Buckley CA, Jacobs JJ (1993) In vivo corrosion of modular hip prosthesis components in mixed and similar metal combinations. The effect of crevice, stress, motion, and alloy coupling. J Biomed Mater Res 27:1533-1544

8. Jacobs JJ, Gilbert JL, Urban RM (1998) Corrosion of metal orthopaedic implants. J Bone Joint Surg Am 80:268-282

9. Katti K (2004) Biomaterials in total joint replacement. Colloids Surf Biointerfaces 39:133-142

10. Mathew MT, Jacobs JJ, Wimmer MA (2012) Wear-corrosion synergism in a CoCrMo hip bearings. Clin Orthop Relat Res 470:3109-3117

11. Mathew MT, Kerwell S, Lundberg HJ, Sukotjo C, Mercuri LG (2014) Tribocorrosion and oral and maxillofacial surgical devices. Br J Oral Maxillofac Surg 52:396-400 